\title{
Efeitos térmicos e mecânicos da superfície na geração e dissipação de Energia Cinética Turbulenta em uma Camada Limite Convectiva
}

\author{
Thermal and mechanical effects of surface in the generation and dissipation of \\ Turbulent Kinetic Energy in a Convective Boundary Layer
}

\author{
João Marcelo Hoffmann Souza*1 e Andre Becker Nunes² \\ ${ }^{1}$ Graduando em Meteorologia, Faculdade de Meteorologia, Universidade Federal de Pelotas, Pelotas, Brasil \\ ${ }^{2}$ Doutor em Meteorologia, Universidade Federal de Pelotas, Pelotas, Brasil
}

\begin{abstract}
Resumo
O fluxo de calor sensível da superfície $\left(H_{s}\right)$ e a rugosidade superficial (zo) são efeitos térmicos e mecânicos, respectivamente, que influenciam na quantidade de turbulência da camada limite planetária (CLP) - tanto na geração como na dissipação da turbulência. A variável que pode quantificar esta turbulência é a energia cinética turbulenta, que tem seu ciclo diurno determinado pela variação horária do fluxo de calor sensível. Sendo a turbulência constituída por uma hierarquia de vórtices, a energia cinética turbulenta total (ECT) pode ser dividida em energia dos grandes vórtices e a energia dos pequenos vórtices (e), cuja principal função é dissipar a energia dos vórtices maiores. Neste trabalho, foi analisada, por meio de modelagem analítica, a influência da rugosidade superficial na geração e dissipação de ECT durante uma camada limite matutina. Os resultados mostram que a ECT e sua dissipação são mais intensas próximas à superfície. Também se observou que quanto maior zo maior a dissipação de energia. Para qualquer período dentro da fase de transição matutina foi encontrado que "e" corresponde a $20 \%$ de ECT independente do tipo de rugosidade. Por último, foi observado que a variação temporal da dissipação é maior quanto menor for zo.
\end{abstract}

Palavras-chave: Transição matutina, fluxo de calor sensível, comprimento de rugosidade, escala de subgrade, grandes vórtices.

\begin{abstract}
The surface sensible heat flux $\left(H_{s}\right)$ and roughness $\left(z_{0}\right)$ are thermal and mechanical effects, respectively, that influence the amount of turbulence into the planetary boundary layer - both in generation and in dissipation of turbulence. The key that may quantify this turbulence is the turbulent kinetic energy, whose diurnal cycle is determinate by the time variation of sensible heat flux. The turbulence being formed by a hierarchy of eddies, the total turbulent kinetic energy (TKE) can be divided into large eddies energy and small eddies energy $(e)$, which the main function is dissipate the energy of the largest. By means of analytical modeling, in this work was analyzed the influence of surface roughness on the generation and dissipation of TKE during a morning boundary layer. The results show ECT and dissipation rate more intense near the surface. We also observed that the larger zo the larger dissipation of energy. At any time within the morning transition phase has been found that " $e$ " represents $20 \%$ of ECT regardless the roughness. Finally, we noted that the larger the time variation of dissipation the smaller zo.
\end{abstract}

Keywords: Morning transition, sensible heat flux, roughness length, subgrid scale, large eddies.

*jmtodos@yahoo.com.br

Recebido: 31/01/2014 Aceito: 22/04/2014 


\section{Introdução}

$\mathrm{P}$ or ser a camada mais baixa da troposfera, a Camada Limite Planetária (CLP) sofre a ação direta da superfície, o que a torna tipicamente turbulenta. O estudo da turbulência tem grande importância para a compreensão dos processos físicos que ocorrem na CLP uma vez que além de interferir diretamente no comportamento de fenômenos de escala maior (ARYA, 1977; ANTHES, 1983) estes também atuam em menores escalas como, por exemplo, na dispersão de poluentes (PONDERGRASS; ARYA, 1984; SOFIEV et al., 2010). A turbulência pode ser definida como movimentos irregulares de redemoinhos denominados vórtices, os quais são gerados principalmente por forçantes da superfície como o fluxo de calor, atrito e obstáculos. O escoamento turbulento é caracterizado por um número elevado de vórtices com dimensões variadas contribuindo para o transporte de momentum, calor e massa na CLP. Essas interações se devem em parte à intensidade da energia cinética turbulenta (ECT) dos vórtices presentes no escoamento. Sabendo que a turbulência é constituída por uma hierarquia de vórtices, temos que a ECT pode ser dividida entre a energia dos grandes vórtices e a energia dos pequenos vórtices (e), também chamados de vórtices de subgrade (DEGRAZIA et al., 2007).

A superfície terrestre - bem como suas características, como a rugosidade aerodinâmica $(z o)$ - está diretamente ligada com a formação de turbulência na CLP. De acordo com Wieringa (1992) os parâmetros de rugosidade descrevem o quanto uma superfície é efetiva em transformar a energia do vento médio sobre ela em movimento turbulento. Outro parâmetro importante no estudo da turbulência na CLP é o fluxo de calor na superfície $\left(H_{s}\right)$. Este parâmetro é função da radiação solar incidente e das características da superfície como tipo de cobertura e de solo (PLEIM; XIU, 1995; STULL, 1988). A variação temporal de Hs controla o ciclo diurno da ECT na CLP. Nesse contexto, o presente trabalho tem como objetivo analisar as diferenças da ECT, durante uma transição matutina, para diferentes coberturas superficiais, i.e., diferentes comprimentos de rugosidade.

\section{Modelagem analítica}

Aqui, a análise da influência de zo na energia cinética turbulenta de subgrade (e) emprega a equação de Nunes et al. (2010):

$$
e=\left[0.65-0.33\left(0.004+\frac{Z}{z_{i}}\right)^{\frac{1}{2}}\right]\left[c 1 u_{*}^{2}+c 2 w_{*}^{2}\right]
$$

Onde $z$ é uma altura acima do solo e $z i$ a altura da camada limite convectiva. Os parâmetros $c 1$ e $c 2$ são dados por:

$$
\left[\begin{array}{l}
c 1 \\
c 2
\end{array}\right]=\left[\begin{array}{c}
{\left[-2,4\left(\frac{z}{z i}\right)^{2}+3,7\left(\frac{z}{z_{i}}\right)+0,65\right]^{-1}} \\
0.075 c 1
\end{array}\right]
$$

A velocidade de fricção $u^{*}$ é obtida da relação do vento na camada superficial, dada por Stull (1988):

$$
\frac{\bar{u}}{u_{*}}=\frac{1}{k} \ln \frac{z}{z_{0}}
$$

e a velocidade de escala convectiva de acordo com Deardorff (1970):

$$
w_{*}=\left[\frac{g}{\bar{T}} Z_{i} H_{S}\right]^{\frac{1}{3}}
$$

Onde g é a aceleração da gravidade, $\bar{T}$ a temperatura média na camada e $H_{s}$ o fluxo de calor sensível na superfície. A energia cinética turbulenta total (ECT) é obtida das componentes das variâncias da velocidade (ŠAVLI, 2012):

$$
E C T=\frac{1}{2}\left(\sigma_{u}^{2}+\sigma_{v}^{2}+\sigma_{w}^{2}\right)
$$

com $\sigma_{u}^{2}, \sigma_{v}^{2}$ e $\sigma_{w}^{2}$ sendo as componentes zonal, meridional e vertical, respectivamente, da variância da velocidade turbulenta obtidas de Degrazia et al. (1997):

$$
\frac{\sigma_{i}^{2}}{w_{*}^{2}}=1.07 c_{i}\left(\frac{z}{z_{i}}\right)^{\frac{2}{3}}\left(\frac{\psi}{\left(f_{m}^{*}\right)_{i}^{c}}\right)^{\frac{2}{3}}
$$

Onde $c_{i}=0.3,0.4,0.4$ nas direções $i=u, v, w$ respectivamente, $\left(f_{m}^{*}\right)_{i}^{c}$ é a frequência espectral do pico de energia (eq. 7) ambos obtidos de Degrazia et al. (2001). A função de dissipação não dimensional $\psi$ é dada pela eq. $10 \mathrm{com}$ a taxa de dissipação média $\varepsilon$ da ECT (eq. 11) obtida de Deardorff (1980).

$$
\begin{aligned}
& \left(f_{m}^{*}\right)_{w}^{c}=0,55\left(\frac{z}{z_{i}}\right)\left[1-\exp \left(-\frac{4 z}{z_{i}}\right)-\right. \\
& \left.0,0003 \exp \left(\frac{8 z}{z_{i}}\right)\right]^{-1}
\end{aligned}
$$




$$
\begin{aligned}
& \psi=\varepsilon Z_{i} / W_{*}^{3} \\
& \varepsilon=C_{S}\left(e^{3 / 2} / l\right)
\end{aligned}
$$

Será utilizado o comprimento de mistura para os pequenos vórtices, $l=50 \mathrm{~m}$, calculado por Degrazia et al. (2007) e a constante $C S=0,93$ para o caso convectivo (MOENG e WYNGAARD, 1988).

\section{Metodologia}

A análise foi realizada para diferentes rugosidades superficiais; tais comprimentos de rugosidade aerodinâmicos respeitaram a classificação de Davenport (WIERINGA, 1992), conforme a Tabela abaixo.

Tabela 1. Classificação do terreno quanto ao comprimento de rugosidade. Adaptado de Wieringa (1992).

\begin{tabular}{l|c}
\hline Descrição do terreno & $\mathbf{z}_{\mathbf{0}}(\mathrm{m})$ \\
\hline Mar aberto & 0,0002 \\
\hline Terrenos planos, sem vegetação, neve, sem obstáculos & 0,005 \\
\hline Terreno plano aberto, grama, obstáculos isolados & 0,03 \\
\hline Culturas baixas, grandes obstáculos isolados & 0,1 \\
\hline Culturas altas, obstáculos espalhados & 0,25 \\
\hline Parque, arbustos, inúmeros obstáculos & 0,5 \\
\hline $\begin{array}{l}\text { Cobertura regular de grandes obstáculos (florestas regulares ou } \\
\text { cidades homogêneas) }\end{array}$ & 1 \\
\hline Centro da cidade com edifícios altos e baixos & $\geq 2$ \\
\hline
\end{tabular}

Para cada comprimento de rugosidade da Tab. 1 serão aplicadas as eq. (1) e (5) em três níveis verticais: $z_{1}=100 \mathrm{~m}$, camada superficial; $z_{2}=500 \mathrm{~m}$, camada de mistura; $z_{3}=900 m$, próximo ao topo da CLP na zona de entranhamento. O objetivo aqui é obter o comportamento energético de cada camada variando apenas o parâmetro $z_{0}$, obtendo um perfil vertical de e para cada $z_{0}$.

Para se verificar a variação vertical e temporal de $e$ e ECT ao longo da transição matutina, escolheu-se dois comprimentos de rugosidade. $\mathrm{O}$ primeiro, $z_{0}=$ $0,1 m$, representando superfícies de área cultivada com culturas baixas e de cobertura regular. O segundo, zo $=1 \mathrm{~m}$, representando superfícies regulares de grandes obstáculos e com tamanhos aproximados como florestas regulares ou cidades homogêneas. A camada a ser analisada, $z / z_{i}=0,1$, foi escolhida de acordo com a atuação e presença dos pequenos e grandes vórtices. Este nível vertical está situado na interface entre a camada limite superficial, região da CLP onde os pequenos vórtices têm maior influência, e a camada de mistura, região onde os grandes vórtices são os principais responsáveis pelas características da camada. Para obter a variação temporal da ECT vamos utilizar a análise feita por Nunes (2008) em que foi considerada a variação do fluxo de calor na superfície como constante para a fase de transição matutina. Assim, a variação temporal da ECT será obtida variando $H s$ linearmente, partindo de $0.04 \mathrm{~K} \mathrm{~m} \mathrm{~s}^{-1}$ (relativo às $7 \mathrm{~h}$ da manhã), até o valor máximo de 0.24 $\mathrm{K} \mathrm{m} \mathrm{s}^{-1}$ (relativo às $12 \mathrm{~h}$ ). Após atingir o valor máximo, o fluxo permanece constante pelo período relativo à 3 hara que represente a convecção bem estabelecida.

Já para a estimativa da dissipação da ECT, será usada a eq. (9) para diferentes comprimentos de rugosidade.

\section{Resultados}

Nos três níveis verticais estudados, $e$ apresentou um crescimento logarítmico com relação ao aumento da rugosidade. Os resultados mostram que a camada superficial foi mais influenciada por $z_{0}$ do que as outras (Figura 1), com e representado por e1. Nota-se que em baixos valores de $z$ o qualquer variação na rugosidade implica em grande variação de energia se comparado com superfícies de alto $z_{0}$ onde as taxas são menores. Os perfis de $e$ na camada de mistura e na zona de entranhamento estão representados por $e 2$ e $e 3$ respectivamente.

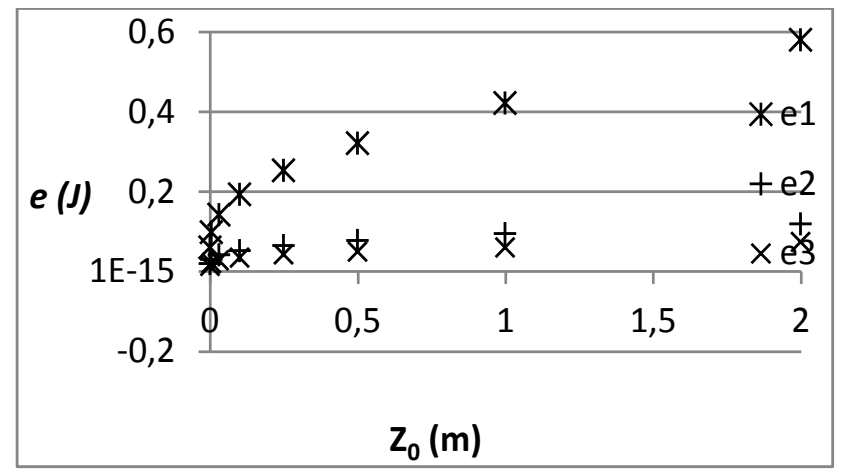

Figura 1. Variação de $e$ com relação a zo para a camada superficial e1, de mistura e2 e zona de entranhamento e3.

O perfil vertical de e para cada z_0 é mostrado na Fig. 2. De $z=0$ até $100 \mathrm{~m}$ os dados foram plotados em intervalos de $10 \mathrm{~m}$, e de $\mathrm{z}=100$ até $1000 \mathrm{~m}$ foram plotados a cada $100 \mathrm{~m}$. Variações significativas de e são observadas em até 500m de altitude. Já na metade superior da CLP a energia varia muito pouco e os valores de e são muito próximos mesmo para as regiões mais energéticas como em superfícies de alta rugosidade mostrando que e influencia muito pouco na camada de mistura. 


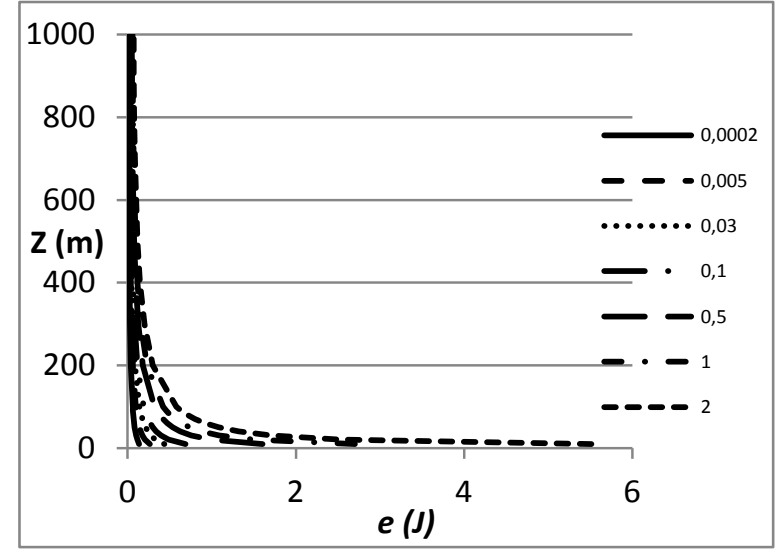

Figura 2. Perfil vertical de e para cada comprimento de rugosidade.

A variação temporal de ECT e $e$, para cada comprimento de rugosidade, é mostrada na Figura 3, em que $e 1$ e ECT1 foram calculados para $z 1_{0}(0.1 \mathrm{~m})$ e $e 2$ e ECT2 foram calculados para $z 2_{0}(1 \mathrm{~m})$.

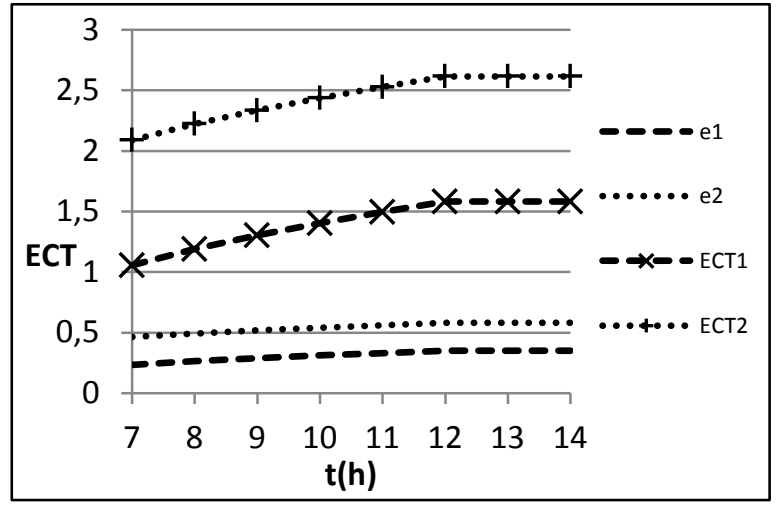

Figura 3. Variação temporal da ECT (J) durante a transição matutina.

Os resultados mostram que quanto maior for $z_{0}$ maior é ECT e e. Além disso, observa-se que mesmo alterando $z_{0}$ a contribuição de $e$ para a ECT permanece constante durante todo o período ( $e 1 / E C T 1=e 2 / E C T 2=0,2216)$.

A Fig. 4 apresenta o comportamento da dissipação para quatro valores de comprimento de rugosidade, $z_{0}$ $=0.005,0.1,1,2 \mathrm{~m}$, e duas situações podem ser destacadas. A primeira corresponde ao decaimento acentuado de $\varepsilon$ ao longo da camada limite superficial $(z \leq 100 m)$. Como mostrado na Fig. 4, as regiões localizadas sobre superfícies de maior $z_{0}$ dissipam mais energia do que as localizadas sobre regiões de menores $z_{0}$, porém tem decaimento de $\varepsilon$ com a altura mais elevado. A segunda situação corresponde ao perfil de $\varepsilon$ na metade superior da CLP $(z \geq 500 m)$. Observa-se que os perfis estão praticamente sobrepostos indicando indiferença de $\varepsilon$ entre todos os valores de $z_{0}$ com dissipação praticamente nula e constante com a altura. Ainda pode ser observado que independente de $z_{0}$ as maiores taxas de dissipação ocorrem próximas à superfície.

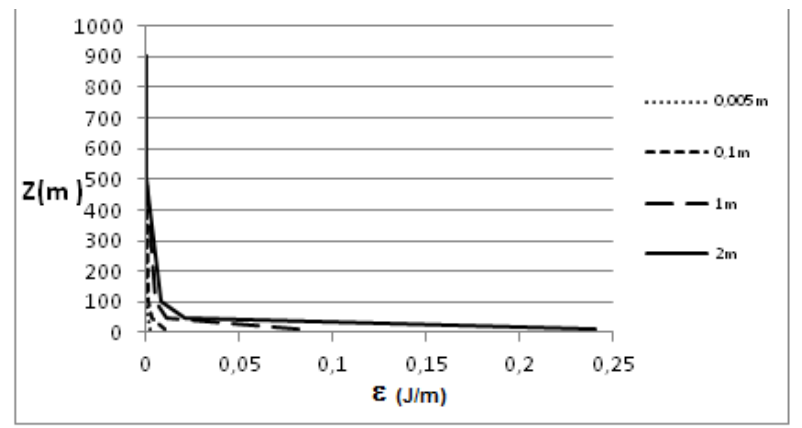

Figura 4. Perfis verticais de $\varepsilon$ para os comprimentos de rugosidade de $0.005 \mathrm{~m}, 0.1 \mathrm{~m}, 1 \mathrm{~m}$ e $2 \mathrm{~m}$.

Como esperado, a maior parte da energia dissipada ocorre na Camada Limite Superficial (CLS). A Fig. 5 mostra o comportamento de $\varepsilon$ ao longo de cinco níveis verticais inseridos na CLS, $z=10,25,50,75,100 m$. Aqui fica evidente a função desempenhada por $z_{0}$ na dissipação de ECT em cada nível. Enquanto é observado um aumento exponencial de $\varepsilon$ com aumento de $z_{0}$ à $10 \mathrm{~m}$ acima da superfície, essa relação tende à linearidade em níveis mais altos.

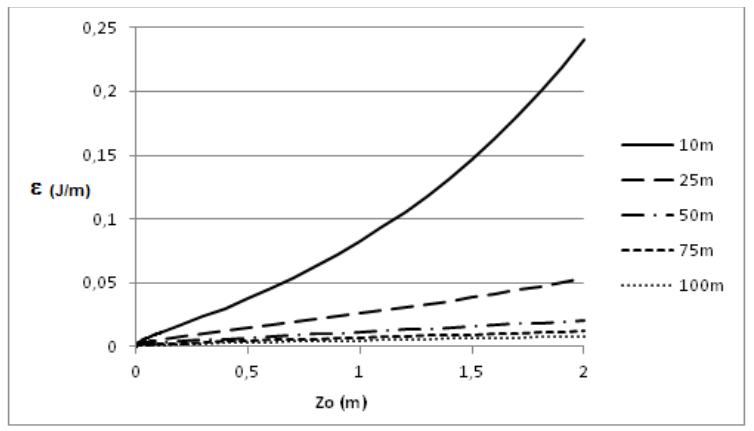

Figura 5. $-\varepsilon$ em função de $z_{0}$ para $z=10,25,50,75$ e $100 \mathrm{~m}$.

Para entender como $z_{0}$ influencia na variação temporal de $\varepsilon$ foi calculado um índice taxa de dissipação de ECT através da razão entre $\varepsilon$ às $7 \mathrm{~h}$ e às $12 \mathrm{~h}$ mostrados na Fig. 6 para os níveis verticais $z=10,50,100 \mathrm{~m}$. O primeiro ponto a ser observado é que nos níveis mais altos da CLS $\varepsilon$ é mais sensível à variação temporal. O segundo ponto observado é que as regiões acima dos menores $z_{0}$ apresentaram as maiores variações temporais de $\varepsilon$ com taxa de dissipação às $7 \mathrm{~h}$ representando $72 \%$ do valor encontrado para às $12 \mathrm{~h}$ em $z=100 \mathrm{~m}$ e $79 \%$ em $z=10 \mathrm{~m}$. Para os maiores $z_{0}$ esse valor chega a $96 \%$ em $z=100 \mathrm{~m}$ e $99 \%$ em $z=10 \mathrm{~m}$. Esses resultados mostram a predominância do termo de produção térmica sobre o de produção mecânica de ECT em uma CLC bem estabelecida acima dos pequenos 
zo. Foi observada pouca variação das $12 \mathrm{~h}$ s com relação as 7hs para níveis mais altos, longe do contorno solido que provoca mais dissipação.

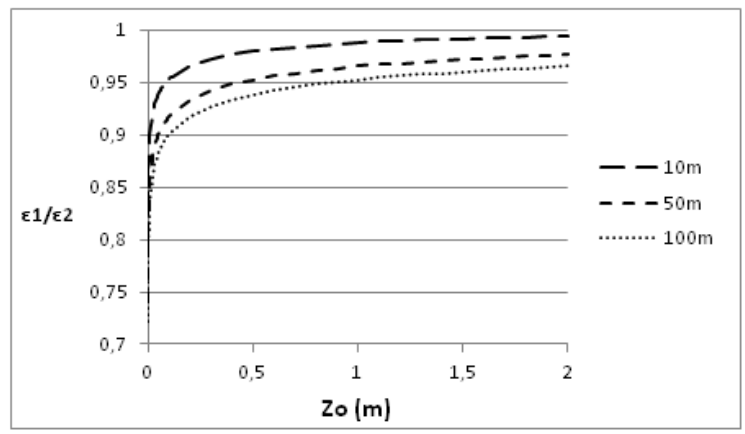

Figura 6. Razão de dissipação para a CLS matutina em função da rugosidade.

\section{Conclusões}

Por meio de modelagem analítica, foi observado que a ECT de subgrade sofre as maiores variações com zo próximo à superfície enquanto que na metade superior da CLP é praticamente constante. Pode-se ainda concluir que superfícies de maior $z_{0}$ dissipam mais energia e a maior parte desta dissipação ocorre na camada superficial evidenciando a relação entre e $e$ a superfície.

Os resultados também mostram que a ECT (tanto total quanto para os pequenos vórtices) é diretamente proporcional à rugosidade da superfície. Além disso, pode-se concluir que, independente da rugosidade, $e \approx$ 0,2 ECT, enquanto o restante da energia é devido à ação dos grandes vórtices.

Por fim, o papel da superfície terrestre na dissipação de ECT na CLP foi estudado. Independente das características superficiais, a dissipação de ECT ocorreu na metade inferior da CLP intensificando-se com a proximidade da superfície. Ainda, verificou-se que a variação matutina de $\varepsilon$ claramente visível na presença dos pequenos $z_{0}$ tornou-se praticamente inexistente para os maiores valores deste parâmetro.

\section{Referências}

ANTHES, R. A. Regional Models of the Atmosphere in Middle Latitudes. Monthly Weather Review, v. 111, p. 1306-1335, 1983.

ARYA, S. P. S. Suggested Revisions to Certain Boundary Layer Parameterization Schemes used in Atmospheric Circulation Models. Monthly Weather Review, v. 105, p. 215-227, 1977.

DEARDORFF, J. Convective velocity and temperature scales for the unstable planetary boundary layer and Rayleigh convection. Journal of the Atmospheric Sciences, v. 27, p. 1211-1213, 1970.

DEARDORFF, J. W.; WILLIS, G. E.; STOCKTON, B. $\mathrm{H}$. Laboratory studies of the entrainment zone of a convectively mixed layer. Journal of the Atmospheric Sciences, v. 100, p. 41-64, 1980.

DEGRAZIA, G. A.; CAMPOS VELHO, H.; CARVALHO, J. Nonlocal exchange coefficients for the convective boundary layer derived from spectral properties. Beitraege zur Physik der Atmosphaere, v. 70, p. 57-64, 1997.

DEGRAZIA, G. A.; DAVIDSON, M. M.; VILHENA, M. T. Derivation of an Eddy Diffusivity Depending on Source Distance for Vertically Inhomogeneous Turbulence in a Convective Boundary Layer. Journal of Applied Meteorology, v. 40, P. 12331240, 2001.

DEGRAZIA, G. A.; NUNES, A. B.; SATYAMURTY, P.; ACEVEDOA, O. C.; VELHO, H. F. D. C.; RIZZA, U.; CARVALHO, J. C. Employing Heisenberg's turbulent spectral transfer theory to parameterize sub-filter scales in LES models. Atmospheric Environment, v. 41, p. 7059-7068, 2007.

MOENG, C-H; WYNGAARD, J. Spectral analysis of large-eddy simulations of the convective boundary layer. Journal of the Atmospheric Sciences, v. 45, p. 3573-3587, 1988.

NUNES, A. B. Crescimento da camada limite convectiva: estudo analítico e numérico. 2008, 190 p. Tese (Doutorado em Meteorologia) - INPE, São José dos Campos

NUNES, B.; CAMPOS VELHO, H. F.; SATYAMURTY, P.; DEGRAZIA, G.; GOULART, A.; RIZZA, U. Morning Boundary-Layer Turbulent Kinetic Energy by Theoretical Models. Boundary-Layer Meteorology, v. 134, p. 23-39, 2010.

PLEIM, J.; XIU, A. Development and testing of a surface flux and planetary boundary layer model for application in mesoscale models. Journal of Applied Meteorology , v. 34, p. 16-32, 1995.

PONDERGRASS, W. R.; ARYA, S. P. S. Dispersion in Neutral Boundary Layer Over a Step Change in Surface Roughness - Part II: Concentration Profiles and Dispersion Parameters. Atmospheric Environment, v. 18, p. 1281-1296, 1984.

ŠAVLI, M. Disponível em: <http://mafija.fmf.unilj.si/ seminar/files/2011_2012/MaticSavli_2.pdf>. Acesso 
em: 01 set. 2013.

SOFIEV, M.; GENIKHOVICH, E.; KERONEN, P.; VESALA, T. Diagnosing the Surface Layer Parameters for Dispersion Models within the Meteorological-to-Dispersion Modeling Interface. Journal of Climate and Applied Meteorology, v. 49, p. 221-233, 2010.

STULL, R. B. An introduction to boundary layer meteorology. Dordrecht: Kluwer Academic Publishers, 1988. 666p.

WIERINGA, J. Updating the Davenport roughness classification. Journal of Wind Engineering and Industrial Aerodynamics, v. 41, p. 357-368, 1992. 